\title{
EDUCAÇÃO INTEGRAL EM ÂMBITO NACIONAL: RESULTADOS DE UM LEVANTAMENTO BIBLIOGRÁFICO
}

Alana Paula de Oliveira, Raquel Pozzenato Silazaki, Samanta Antunes Kasper, Adriana Locatelli França, Renata Portela Rinaldi

Universidade Estadual Paulista - UNESP, Programa de Pós-Graduação em Educação, Presidente Prudente, SP. E-mail: alanapaulla@hotmail.com

\section{RESUMO}

Este trabalho é decorrente de uma pesquisa em rede intitulada "Rede de Pesquisa e Formação sobre Educação Integral: experiências, movimentos, inovação e desafios contemporâneos" que congrega pesquisadores de cinco universidades públicas - estaduais e federais - das regiões sudeste, sul, centro-oeste e norte do Brasil e da cidade de Barcelona, Espanha. Visa fomentar as discussões e o conhecimento acerca da educação integral, educação em tempo integral e escolas em período integral ou jornada ampliada e investigar a implementação da jornada ampliada do Sistema Municipal de Ensino de Presidente Prudente. A partir do levantamento bibliográfico de artigos no Portal de Periódicos Capes, entre 2007 a 2018, com os descritores: Educação Integral, Educação em Tempo Integral, Escola de Tempo Integral, objetivamos identificar como as experiências e a política pública de/para educação integral se desenvolve ao longo dos últimos anos no Brasil e no exterior.

Palavras-chave: educação integral, educação em tempo integral, escola de tempo Integral, escola com jornada ampliada, pesquisa bibliográfica.

\section{INTEGRAL EDUCATION IN NATIONAL SPHERE: RESULTS OF A BIBLIOGRAPHIC RESEARCH}

\begin{abstract}
This study is the result of a network research entitled "Research and Training Network on Integral Education: Experiences, Movements, Innovation and Contemporary Challenges" that brings together researchers from five public universities - state and federal - from the Southeast, South, Midwest and northern Brazil and the city of Barcelona, Spain. It aims to foster discussions and knowledge about integral education, full-time education, full-time schools or extended school day and investigate the implementation of the full-time schools of the Teaching System Municipality of Presidente Prudente. Based on a bibliographic research of articles in the Capes Periodicals Portal, between 2007 and 2018, with the descriptors: Integral Education, Full-Time Education, Full-Time Schools, we aim to identify how the experiences and the public policy of / for integral education are developed over the last years in Brazil and abroad.
\end{abstract}

Keywords: integral education, full-time education, full-time schools, extended school day, bibliographic research. 


\section{INTRODUÇÃO}

Torna-se cada vez mais imprescindível propor o debate da Educação Integral no país, pois coaduna com a necessidade dos diferentes entes federados promovê-la, em regime de colaboração, visando consolidar o direito à educação, o qual o indivíduo detém. É urgente e necessário ampliar o conhecimento acerca deste importante conceito (Educação Integral), visto que construir condições para sua concretização, requer um compromisso não só dos governos (federal, estadual e municipal) como da sociedade acadêmica, dos gestores e professores em suas distintas realidades e da comunidade de modo geral.

Tal processo que exige ampla participação, requer o constante repensar sobre a escola pública, pois são inúmeros os desafios que são colocados a ela. Nos últimos tempos, temos observado e nos deparado com alunos que estão permanentemente conectados e chegam às escolas com um universo considerável de informações. Vivem num mundo tecnológico e repleto de mídias que consomem fortemente a atenção dos indivíduos, exigindo que a escola acompanhe essa amplitude advinda dos avanços e desenvolvimento tecnológicos, inerentes a essa sociedade globalizada. Suas interfaces, benefícios e malefícios circulam dentro dos muros escolares responsabilizando a escola, cada vez mais, a oferecer um ensino que permita a este aluno ter consciência de sua ação/atuação em sociedade, mas, acima de tudo, construir conhecimentos que Ihe permitam agir criticamente mediante às inúmeras adversidades e desafios que estão presentes no cotidiano.

Este repensar a escola, exige considerar o que já temos construído sobre ela, pois há toda uma malha teórica, embasamentos históricos, filosóficos, pedagógicos etc., que permitiram que ela se desenvolvesse até aqui. Entretanto, temos visto a cada dia que a educação tem exigido considerar o que as novas gerações, sob suas condições singulares, necessitam para a consolidação de uma educação integral.

Neste sentido, o objetivo deste estudo é apresentar, a partir de um levantamento bibliográfico realizado no Portal de Periódicos da Coordenação de Aperfeiçoamento de Pessoal de Nível Superior (Capes), a incidência de produções decorrentes ao longo do período de 2007 a 2018, que tratam da temática da educação integral, a partir dos descritores: Educação Integral, Educação em Tempo Integral e Escolas de Tempo Integral, haja vista a implantação de políticas públicas educacionais, sobretudo, nas últimas décadas, que lançaram às escolas a possiblidade de ampliarem suas jornadas para a oferta de uma educação integral.

Como temos observado as diferentes formas de olhar à questão, inclusive sobre a celeuma acerca dos termos que foram pesquisados na base de dados da Capes, pela qual ainda é visível uma certa complexidade para delimitá-los e conceituá-los, visamos olhar para a temática. Haja vista, que independentemente da ampliação das jornadas, a educação integral em sua abordagem conceitual, deve se concretizar superando a diferentes implicações desses tempos e, também dos espaços que a escola venha a oferecer.

A partir da pesquisa em rede intitulada "Rede de Pesquisa e Formação sobre Educação Integral: experiências, movimentos, inovação e desafios contemporâneos", coordenada por Rinaldi (2017) que congrega pesquisadores ${ }^{1}$ de cinco universidades públicas - estaduais e federais - das regiões sudeste, sul, centro-oeste e norte do Brasil e da cidade de Barcelona, Espanha, temse como principal objetivo identificar, conhecer e analisar como as experiências e a política pública de/para educação integral se desenvolve ao longo dos últimos anos no Brasil e no exterior.

Especificamente para o presente texto, temos como objetivo identificar as diferentes experiências desenvolvidas acerca da Educação Integral no país a partir das produções científicas em periódicos disponíveis no Portal de Periódicos da Capes. Foi proposta uma dinâmica de trabalho coletivo que, a partir do levantamento bibliográfico na base de dados supramencionadas,

\footnotetext{
${ }^{1}$ Além dos pesquisadores, fazem parte do grupo de investigação estudantes de Programa de Pós-graduação em Educação, doutorado e mestrado acadêmico e profissional -, de graduação, profissionais e professores da educação básica. 
permitiu que os envolvidos (discentes da graduação, do mestrado e doutorado) desenvolvessem coletivamente um trabalho que alinhou a colaboração, a interação e o compromisso permitindo ultrapassar as formas de pensar e agir individual, na busca por trabalhos que irão, no decorrer da pesquisa em desenvolvimento, ampliar o conhecimento acerca da temática da educação integral, além disso propor reflexões acerca das formas e intenções da implantação e implementação dessa política no país.

\section{METODOLOGIA}

O presente trabalho está apoiado pela pesquisa qualitativa que nos permite uma visão ampliada dos fenômenos educacionais, sobretudo a partir da perspectiva das políticas públicas educacionais que envolvem a educação integral. Porém, valeu-se de dados quantitativos acerca das produções que foram levantadas e que permitirão, num próximo momento da pesquisa, aproximar os dados para análises mais aprofundadas, a fim de observar suas diferentes intercorrências no campo da educação integral em jornadas ampliadas.

Acerca de um trabalho sobre estudos quantitativos realizado por Gatti (2004), a autora nos ensina que:

Os métodos de análise de dados que se traduzem por números podem ser muito úteis na compreensão de diversos problemas educacionais. Mais ainda, a combinação deste tipo de dados com dados oriundos de metodologias qualitativas, podem vir a enriquecer a compreensão de eventos, fatos, processos. As duas abordagens demandam, no entanto, o esforço de reflexão do pesquisador para dar sentido ao material levantado e analisado. (GATTI, 2004, p. 13)

Assim, como suporte para este trabalho, os dados quantitativos possibilitarão, futuramente, ao grupo de pesquisadores contextualizar e cotejar com a literatura como foi implantada e como vem sendo implementada a educação integral no país e, sobretudo, possibilitar observar quais dificuldades e desafios ainda estão presentes na sua implementação.

Neste trabalho, apresentamos resultados da primeira fase da pesquisa, que consistiu de um levantamento de artigos completos na base de dados do Portal de Periódicos da Capes, utilizando como critérios de refinamento apenas o "ano", no período de 2007 a 2018, para os descritores "educação integral", "educação em tempo integral" e "escola de tempo integral", de modo a garantir que o maior número possível de trabalhos fosse encontrado e validado para esta investigação.

Tomamos como ponto de partida o ano de 2007, pois com a implantação do Programa Mais Educação, pelo Ministério da Educação, representa um marco na política educacional de indução da educação integral e da ampliação do tempo escolar nas escolas públicas brasileiras.

\section{RESULTADOS}

Foram identificados um total de 9.662 artigos científicos na base de dados investigada, no período determinado. A partir da análise dos títulos, palavras-chave e resumos dos artigos, procuramos selecionar todos os trabalhos com conteúdos relacionados ao objeto de estudo. Conforme podemos visualizar por meio do Quadro 1, foram selecionados para análise na íntegra um total de 129 trabalhos. 
Quadro 1 - Total de artigos selecionados por descritor e período de tempo.

\begin{tabular}{|l|l|l|l|l|l|l|l|l|l|l|l|l|l|}
\hline & $\begin{array}{l}\mathbf{2 0 0} \\
\mathbf{7}\end{array}$ & $\begin{array}{l}\mathbf{2 0 0} \\
\mathbf{8}\end{array}$ & $\begin{array}{l}\mathbf{2 0 0} \\
\mathbf{9}\end{array}$ & $\begin{array}{l}\mathbf{2 0 1} \\
\mathbf{0}\end{array}$ & $\begin{array}{l}\mathbf{2 0 1} \\
\mathbf{1}\end{array}$ & $\begin{array}{l}\mathbf{2 0 1} \\
\mathbf{2}\end{array}$ & $\begin{array}{l}\mathbf{2 0 1} \\
\mathbf{3}\end{array}$ & $\begin{array}{l}\mathbf{2 0 1} \\
\mathbf{4}\end{array}$ & $\begin{array}{l}\mathbf{2 0 1} \\
\mathbf{5}\end{array}$ & $\begin{array}{l}\mathbf{2 0 1} \\
\mathbf{6}\end{array}$ & $\begin{array}{l}\mathbf{2 0 1} \\
\mathbf{7}\end{array}$ & $\begin{array}{l}\mathbf{2 0 1} \\
\mathbf{8}\end{array}$ & $\begin{array}{l}\text { TOTA } \\
\text { L }\end{array}$ \\
\hline $\begin{array}{l}\text { Educaçã } \\
\mathbf{0} \\
\text { integral }\end{array}$ & 1 & 1 & 1 & 3 & 1 & 6 & 6 & 13 & 30 & 19 & 23 & 6 & 110 \\
\hline $\begin{array}{l}\text { Educaçã } \\
\text { o em } \\
\text { tempo } \\
\text { integral }\end{array}$ & 0 & 0 & 2 & 1 & 0 & 2 & 0 & 1 & 1 & 1 & 0 & 0 & 8 \\
\hline $\begin{array}{l}\text { Escola } \\
\text { de } \\
\text { tempo } \\
\text { integral }\end{array}$ & 0 & 1 & 1 & 0 & 1 & 0 & 0 & 6 & 1 & 1 & 0 & 0 & 11 \\
\hline TOTAL & 1 & 2 & 4 & 4 & 2 & 8 & 6 & 20 & 32 & 21 & 23 & 6 & 129 \\
\hline
\end{tabular}

Fonte: Elaboração própria a partir dos resultados do levantamento bibliográfico.

Com o descritor "educação integral", de um total de 4.410 artigos, foram selecionados 110 trabalhos. No que tange ao descritor "educação em tempo integral", foram consultados 2.642 trabalhos, sendo 55 selecionados. Entretanto, 47 trabalhos já haviam aparecido anteriormente no descritor "educação integral", restando assim apenas 8 artigos inéditos. No que se refere ao unitermo "escola de tempo integral", verificou-se um total de 2.610 trabalhos e 41 foram selecionados. Contudo, destes 41 , contatou-se que 30 trabalhos haviam sido previamente verificados nos descritores anteriores e, assim, selecionamos apenas 11 artigos.

O gráfico 1, ilustra os resultados obtidos e nos permite realizar algumas discussões:

\section{Gráfico 1 - TOTAL DE ARTIGOS SELECIONADOS}

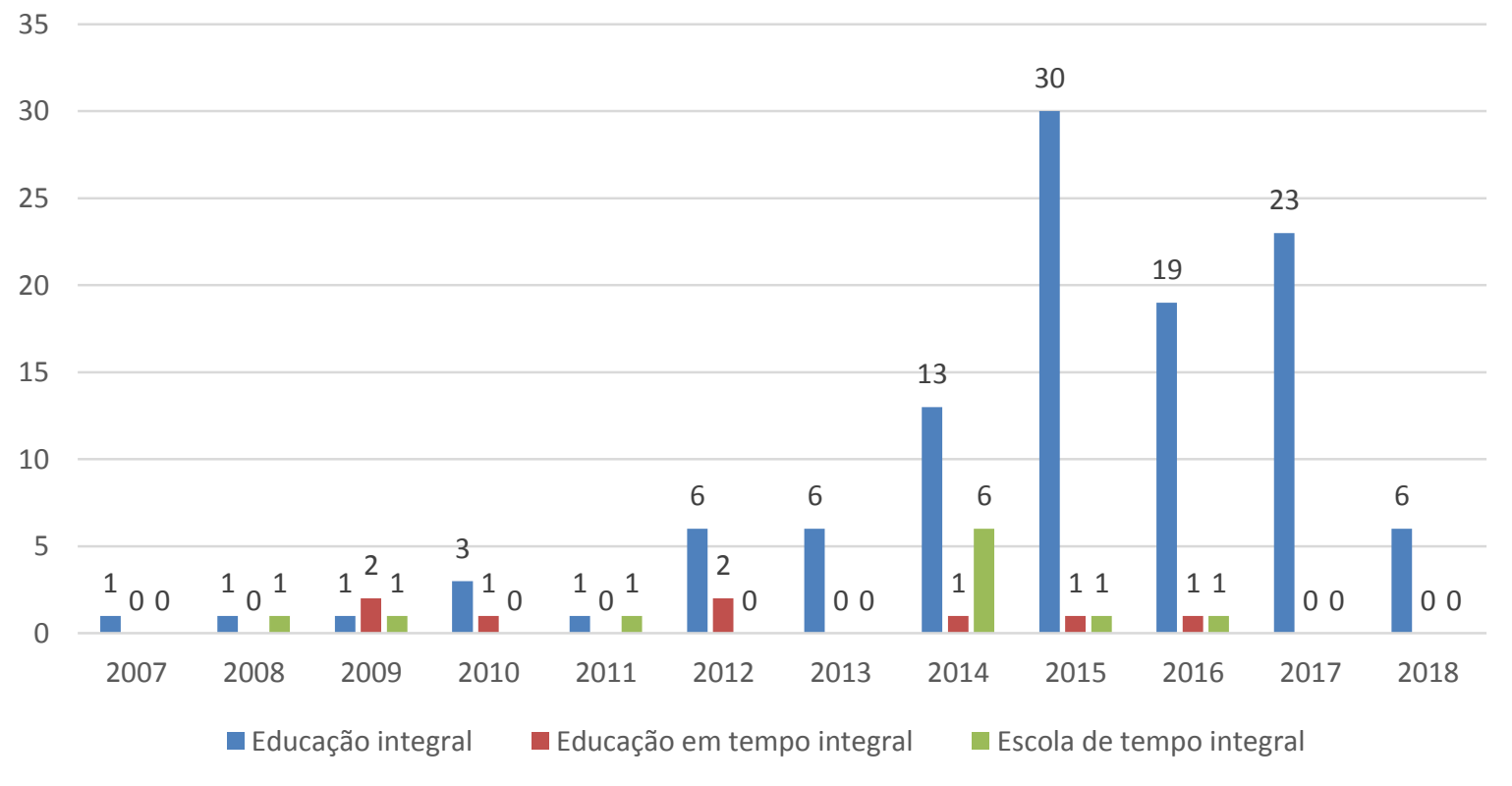

Encontramos um número maior de trabalhos relacionados ao descritor "educação integral", o qual possibilitou, também, um montante maior de trabalhos selecionados. Nota-se que há uma regularidade quanto a investigação da temática ao longo da década. Muitos trabalhos selecionados com este primeiro descritor foram identificados nos demais, porém, somente 
consideramos para análise os trabalhos inéditos, ou seja, aqueles que se repetiam nos demais descritores foram descartados, uma vez que serão analisados na próxima etapa da pesquisa. Podemos notar uma evolução de publicações a partir do ano de 2014. No entanto, identificamos um número significativamente maior de trabalhos publicados no ano de 2015, sendo que, dos 129 artigos selecionados, 32 são deste período.

Vale destacar que o levantamento ocorreu em final do segundo semestre de 2018 e, portanto, outros trabalhos ainda podem ser publicados no corrente ano. Dessa forma, pretendemos realizar uma nova busca assim que o ano se findar atualizando as informações.

\section{DISCUSSÕES}

Diante dos resultados apresentados, podemos afirmar que há uma tendência maior de trabalhos relacionados à educação integral. No entanto, é pertinente questionarmos se realmente tais produções se referem à temática conforme conceitos apresentados por autores e pesquisadores da área.

A educação integral pode ser compreendida como uma formação completa do indivíduo, que ultrapassa a formação cognitiva e o cumprimento dos componentes curriculares. Refere-se à uma educação que contempla os aspectos cognitivo, afetivo, físico, linguístico, político, cultural, moral, ético e estético (CAVALIERE, 2002; COELHO, 2009; PARENTE, 2016; COSTA, 2017).

Nesse sentido, visamos olhar para as produções encontradas com o intuito de descobrir como este conceito tem sido trabalhado nas diferentes localidades deste país e, sobretudo, se as dezenas de experiências encontradas nos permitem, além de observar se o direito à educação tem sido garantido, também observar a forma como este direito está se desenvolvendo para garantir a formação integral do indivíduo. Compreendemos a complexidade que envolve a temática e a garantia de um direito na perspectiva da educação integral. Estamos convencidas de que os dados levantados permitirão ampliar as análises sobre o tema e cotejá-las com a realidade para a qual estamos depositando o olhar, a do Sistema Municipal de Ensino de Presidente Prudente, no intuito de ampliar possibilidades significativas e criar caminhos promissores na perspectiva de uma educação integral.

Cabe salientar que a educação que propomos deve ser integral independe do regime de atendimento, seja de tempo parcial ou integral. Vale ressaltar que essa proposta não pode ser confundida com a escola de tempo integral ou com a educação em tempo integral. A escola de tempo integral se dá a partir de uma política na qual o aluno permanece mais tempo em atividades curriculares que se restringem ao espaço escolar, como salas de aula, laboratórios, salas de vídeo, refeitório, etc. (PARENTE, 2016). Por sua vez, na educação em tempo integral, o aluno divide seu dia entre a escola e outros espaços com atividades diversas (COSTA, 2017). São propostas também enriquecedoras, resta saber como a educação integral é trabalhada nessas diferentes possibilidades educativas, questões a serem averiguadas, neste primeiro momento, nos artigos levantados no Portal de Periódicos da Capes.

\section{CONCLUSÃO}

A educação integral tem sido tema de muitas discussões atuais, porém um olhar atento deve ser debruçado em torno do embasamento teórico-epistemológico destes estudos. No desdobramento desta pesquisa, analisaremos mais profundamente os 129 artigos selecionados a fim de identificar as concepções permeadas nas produções.

O estudo se revela necessário, pois a complexidade do tema é aparente e somado à evolução natural da sociedade que requer, cada vez mais, uma escola que dê respostas para a formação de indivíduos que estão o tempo todo conectados, que vivem em uma sociedade amplamente informatizada/tecnológica e que tem acesso as informações diversas. No entanto, mesmo com toda essa evolução social, tecnológica e globalizada temos uma educação pública que 
ainda é frágil na formação de indivíduos capazes de agirem criticamente e significativamente em sociedade. Todas essas implicações requerem o constante repensar a escola pública, a fim de garantir uma efetiva política que permita, de fato, uma formação integral do indivíduo.

\section{REFERÊNCIAS}

CAVALIERE, A. M. Educação Integral: uma nova identidade para a realidade brasileira? Educ. Soc., Campinas, v. 23, n. 81, 2002, p. 247-270. Disponível em: < http://www.scielo.br/pdf/es/v23n81/13940.pdf>. Acesso em: 18 jul. 2018.

COELHO, L. M. C. História(s) da Educação Integral. In: MAURíCIO, L. V. (Org.). Em Aberto: educação integral e tempo integral, Brasília, v. 22, n. 80, p. 83-96, abr. 2009.

COSTA, R. E. C. A. Ampliação da Jornada Escolar e o Terceiro Setor: a atuação do CENPEC. Educação \& Realidade, Porto Alegre, 2017.

GATTI, B. A. Estudos quantitativos em educação. Educação e Pesquisa, São Paulo, v.30, n.1, p. 1130, jan./abr. 2004. Disponível em: <http://www.scielo.br/pdf/ep/v30n1/a02v30n1.pdf>. Acesso em: 28 jul. 2018.

PARENTE, C. M. D. Construindo uma Tipologia das Políticas de Educação Integral em Tempo Integral. Roteiro, Joaçaba, v. 41, n. 3, p. 563-586, set./dez. 2016.

RINALDI, R. P. Rede de Pesquisa e Formação sobre Educação Integral: experiências, movimentos, inovação e desafios contemporâneos. Projeto de pesquisa. Presidente Prudente: FCT/UNESP, 2017. (mimeo) 